\title{
Características de los pacientes traumatizados que ingresan a la UCI de un hospital general en Chile
}

\author{
CAROLINA RUIZ ${ }^{1,2}$, XIMENA MIMICA ${ }^{1}$, MARÍA LUISA LISBONA ${ }^{1}$, \\ JAVIERA DONOSO ${ }^{3}$, PAULA ARRIAGADA ${ }^{3}$, MACARENA ROA', \\ SEBASTIÁN BRAVO ${ }^{1}$, JORGE GODOY ${ }^{1}$
}

\section{Characteristics of trauma patients admitted to the intensive care unit of a general hospital in Chile}

Background: Trauma is an important cause of death among young adults. Aim: To determine the characteristics, treatments and evolution of trauma patients admitted to an intensive care unit (ICU) of a public hospital in Santiago, Chile. Material and Methods: All polytrauma (PT) and severely traumatized (ST) patients admitted to ICU were included. We recorded the type of trauma along with demographic and hemodynamic variables, treatments and complications. The evolution and treatments received by $P T$ and ST patients were compared. Results: We recorded data from 72 patients aged $43 \pm 21$ years (93\% males). Sixty two percent were PT and $24 \%$ had penetrating injuries. TBI (Trauma Brain Injury) was the most common trauma. On admission, acute Physiology and Chronic Health Evaluation II (APACHE II) score was $18.7+7.3$, and Injury Severity Score (ISS) was $32.8+20.1$. ICU stay was $7.8+6$ days. Sixty seven per cent of patients required surgery and $58 \%$ received blood transfusions. No differences were found between PT and ST. ICU and hospital mortality rates were 15 and 25\% respectively. Conclusions: The characteristics and evolution of PT and ST of this series of patients are similar to those described abroad. Mortality was in agreement with ISS and APACHE II scores.

(Rev Med Chile 2013; 141: 1389-1394)

Key words: Critical Care; Epidemiology; Multiple Trauma.

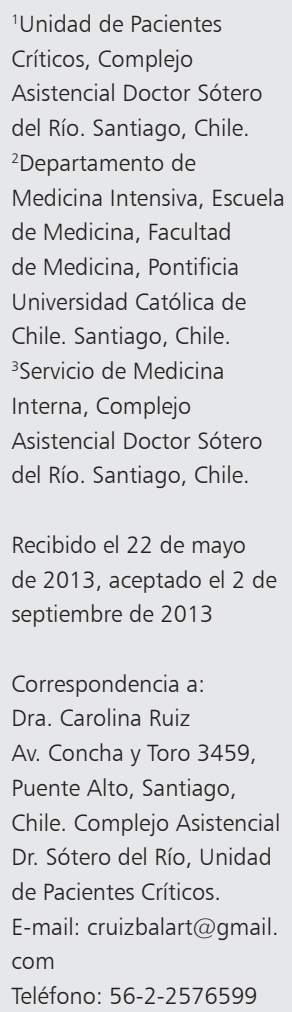

E 1 trauma es la causa más importante de muerte entre los 5 y 44 años, causando $10 \%$ de la mortalidad mundial ${ }^{1}$. Además, debido a las secuelas del trauma, hasta $20 \%$ de los pacientes pueden presentar discapacidad, que es grave en $6 \%$ de los casos. Directa o indirectamente, los traumatismos tienen un alto impacto financiero, por los gastos que generan en atención médica y en ausentismo laboral ${ }^{2,3}$.

Se ha demostrado que la protocolización del manejo del trauma por personal entrenado, incluyendo buenos sistemas de rescate, permite disminuir la mortalidad asociada a este ${ }^{4,5}$. Esto debido a que parte importante de las muertes que se producen durante las primeras horas de evolución (muertes precoces) son prevenibles con un manejo adecuado. Dentro de las causas de muerte precoz por trauma destacan: hemoneumotórax, hematomas cerebrales extraaxiales y lesiones asociadas con sangrado masivo. Además, un manejo protocolizado podría permitir disminuir los días de UCI (Unidad de Cuidados Intensivos) y ventilación mecánica, así como optimizar el uso de recursos como las transfusiones sanguíneas ${ }^{6,7}$.

En este sentido, para poder protocolizar el manejo de una patología es fundamental conocer 
previamente su epidemiología, así como su manejo y evolución. Por el momento, en Chile existen escasos registros de las características y evolución de los pacientes traumatizados que ingresan a una UCI.

El objetivo del presente estudio fue conocer y describir las características, tratamientos recibidos y evolución de los pacientes politraumatizados y traumatizados graves que ingresaron a la UCI del Complejo Asistencial Dr. Sótero del Río durante el año 2011.

\section{Pacientes y Métodos}

Se realizó un estudio descriptivo y prospectivo, incluyendo a todos los politraumatizados y traumatizados graves mayores de 18 años que ingresaron durante el año 2011 a la UCI de adultos del Complejo Asistencial Dr. Sótero del Río. Esta es una UCI polivalente de 18 camas de un hospital general, que atiende aproximadamente un millón y medio de habitantes.

Se clasificó a los pacientes como politraumatizados (PT) y traumatizados graves (TG) de acuerdo a la definición dada por el Ministerio de Salud de Chile ${ }^{4}$. Los PT corresponden a pacientes que presentan lesión en 2 o más sistemas con riesgo vital y los TG a pacientes que presentan lesión en 1 sistema con riesgo vital o riesgo de secuelas importantes.

Se registró el tipo de trauma y las lesiones presentadas por los pacientes. Los pacientes se clasificaron de acuerdo al tipo de trauma predominante en: trauma encéfalo craneano (TEC), trauma raquimedular (TRM), trauma de tórax, trauma abdominal, trauma tóraco-abdominal, trauma vascular y trauma músculo-esquelético. Además, se registraron variables demográficas, hemodinámicas, parámetros de disfunción orgánica, puntajes de gravedad, complicaciones propias del trauma (rabdomiolisis, síndrome compartimental de extremidades, embolia grasa y sangrado), infecciones, cirugías, aporte de fluidos y transfusiones sanguíneas. Se realizó un seguimiento durante los primeros 7 días de hospitalización en UCI o hasta el alta de la UCI (lo que ocurriera primero). Posteriormente, se determinó la condición de alta hospitalaria (vivo o muerto).

Para describir las características de los pacientes y los tratamientos recibidos se utilizó la media y desviación estándar. Además, se comparó la evolución y tratamientos recibidos entre PT y TG, utilizando la prueba t para muestras independientes para variables intervalares y la prueba de $\chi^{2}$ para variables nominales. Se definió un valor de $\mathrm{p}<0,05$ como estadísticamente significativo. Para el análisis estadístico se utilizó el programa IBM SPSS 20.

Este estudio fue aprobado por el Comité de Ética de Servicio de Salud Metropolitano Sur Oriente.

\section{Resultados}

\section{Características generales}

Durante el período de observación ingresaron a la UCI 72 pacientes de trauma, representando $12 \%$ del total de ingresos a la unidad durante el 2011 , con una edad promedio de $42,8 \pm 20,7$ años y en su mayoría hombres (92,7\%). Los pacientes correspondieron a PT en $62,5 \%$, siendo el TEC el tipo de trauma más frecuente, seguido por el trauma músculo-esquelético (Tabla 1). Veinticuatro por ciento de los pacientes presentaron trauma penetrante, correspondiendo en la mayoría de los casos a trauma de tronco (65\%), es decir, trauma de tórax, abdomen, o tóraco-abdominal. En el grupo de pacientes con trauma contuso, la lesión más frecuente fue el TEC (70\%) y sólo 13\% de los pacientes presentó trauma de tronco.

El puntaje APACHE II (Acute Physiology and Chronic Health Evaluation) fue 18,7 \pm 7,3 y del score ISS (Injury Severity Score) 32,8 $\pm 20,1$. Noventa y cinco por ciento de los pacientes requirió ventilación mecánica $(\mathrm{VM})$, con una duración

Tabla 1. Tipos de trauma presentados por los pacientes

\begin{tabular}{|lc|}
\hline Tipo de trauma & Porcentaje/n \\
\hline TEC & $56 / 40$ \\
\hline T. músculo-esquelético & $13 / 10$ \\
\hline T. tórax & $10 / 7$ \\
\hline T. tórax-abdominal & $8 / 6$ \\
\hline T. abdominal & $6 / 4$ \\
\hline T. vascular & $4 / 3$ \\
\hline TRM & $3 / 2$ \\
\hline
\end{tabular}

TEC (traumatismo encefo craneano), TRM (traumatismo raquimedular). 
Tabla 2. Variables hemodinámicas, de perfusión y aporte de fluídos durante los 3 primeros días

\begin{tabular}{|ccccccc|}
\hline Día & PAM (mmHg) & Lactato (mmol/L) & Hcto (\%) & Fluidos (m) & NA (mcg/kg/min) & n/\% pacientes NA \\
1 & $74,4 \pm 15,6$ & $3,7 \pm 3,5$ & $30,6 \pm 8$ & $2.283 \pm 1.460$ & $0,11 \pm 0,1$ & $44 / 61$ \\
2 & $78,1 \pm 13,7,2$ & $2,5 \pm 1,3$ & $29,4 \pm 5,1$ & $2.126 \pm 1.145$ & $0,12 \pm 0,1$ & $41 / 57$ \\
3 & $82,7 \pm 13,4$ & $1,8 \pm 0,8$ & $28,2 \pm 4$ & $1.474 \pm 959$ & $0,10 \pm 0,1$ & $38 / 52$ \\
\hline
\end{tabular}

Presión arterial media (PAM), Hcto (hematocrito), NA (noradrenalina), n (número) /\% (porcentaje) de pacientes que requirieron noradrenalina

promedio de $6 \pm 5$ días. La estadía en UCI fue 7,8 \pm 6 , días. No hubo diferencias en el APACHE II, estadía en UCI y duración de la VM respecto a los otros pacientes que ingresaron a la UCI por otras patologías durante el período de observación.

\section{Variables hemodinámicas y de perfusión}

Los pacientes requirieron noradrenalina durante los 2 primeros días de evolución, $61 \%$ y $57 \%$ de respectivamente, pero en una dosis moderada, acorde con la cantidad de fluidos recibidos y niveles de lactato plasmáticos. Sólo 8 pacientes requirieron un segundo vasopresor o inótropo además de la noradrenalina. La Tabla 2 muestra la evolución de las variables hemodinámicas durante los 3 primeros días.

A pesar de que los pacientes tuvieron un aporte de fluidos moderado, el balance hídrico acumulado al séptimo día fue $7.830 \pm 4.986 \mathrm{ml}$. Veinte por ciento de los pacientes recibieron coloides (almidón) además de cristaloides.

\section{Tratamientos}

Setenta y siete por ciento de los pacientes requirieron cirugía o algún procedimiento, siendo la instalación de captor de PIC (presión intracraneana) el procedimiento más frecuente (17 pacientes). Veintidós por ciento requirió 2 o más cirugías.

Cincuenta y ocho por ciento de los pacientes recibió transfusiones, principalmente durante los 2 primeros días de evolución (se consideran las transfusiones recibidas en urgencia, pabellón y en la UCI). Nueve pacientes $(13,2 \%)$ recibieron transfusión masiva (transfusión de 10 o más unidades de glóbulos rojos en $24 \mathrm{~h}$ ) durante las primeras 24 $\mathrm{h}$ de ingreso al hospital, con un promedio de 16,5 unidades de glóbulos rojos. La Tabla 3 muestra las transfusiones durante los 2 primeros días.

Al tercer día de evolución, sólo 25\% de los pacientes estaban recibiendo nutrición, incremen-
Tabla 3. Transfusiones recibidas durante los 2 primeros días

\begin{tabular}{|ccccc|}
\hline Día & $\begin{array}{c}\text { GR } \\
\text { (unidades) }\end{array}$ & $\begin{array}{c}\text { PFC } \\
\text { (unidades) }\end{array}$ & $\begin{array}{c}\text { Plaquetas } \\
\text { (unidades) }\end{array}$ & $\begin{array}{c}\text { Criop } \\
\text { (unidades) }\end{array}$ \\
\hline 1 & $6,3 \pm 4,3$ & $9,5 \pm 6,3$ & $6,8 \pm 3,2$ & $6,6 \pm 2,6$ \\
2 & $2,3 \pm 0,8$ & $3,2 \pm 1,1$ & $4,8 \pm 0,9$ & $3,5 \pm 3$ \\
\hline
\end{tabular}

GR (glóbulos rojos), PFC (plasma fresco congelado), Criop (crioprecipitados).

tándose al séptimo día a 76\%. La nutrición enteral fue el tipo más frecuentemente aportado.

\section{Evolución y complicaciones}

Todos los pacientes presentaron al menos una disfunción, siendo la evolución del puntaje SOFA (Secuential-related Organ Failure Assessment) durante los 3 primeros días: 7,4 \pm 3,3; 7,3 \pm 4 y 7,2 $\pm 3,5$ respectivamente.

Cincuenta y cuatro por ciento de los pacientes presentaron complicaciones del trauma (rabdomiolisis, embolia grasa, síndrome compartimental de extremidades y sangrado), siendo la rabdomiolisis la más frecuente (38 pacientes). Se definió rabdomiolisis como un aumento en la CK total mayor a 5 veces el valor normal (el valor normal para el laboratorio del hospital es de hasta 130 $\mathrm{UI} / \mathrm{L})$. Once pacientes tuvieron una CK mayor a 5.000, pero sólo 2 presentaron deterioro de la función renal que podría atribuirse a rabdomiolisis. Cincuenta y ocho porciento de los pacientes presentaron infecciones, siendo la neumonía aspirativa el cuadro más frecuente (17 pacientes).

La mortalidad en UCI fue 15\% (11 pacientes), siendo menor que la mortalidad general de la unidad durante el período de observación, que correspondió a 20\%. Las principales causas de muerte fueron el desarrollo de disfunción orgánica múltiple-sepsis (6 pacientes), seguido por TEC ( 4 
pacientes) y sangrado no controlado (1 paciente). La mortalidad hospitalaria total de los pacientes correspondió a $25 \%$.

\section{Comparación entre politraumatizados y traumatizados graves}

Durante el período de observación ingresaron 45 PT y 27 TG. Al comparar ambos grupos no se encontró diferencia en edad, APACHE II, ISS ni SOFA (SOFA durante los 3 primeros días). Tampoco se encontró diferencia en días de UCI, días de VM, necesidad de cirugía/procedimientos, y sobrevida de UCI. La Tabla 4 compara las variables hemodinámicas de ambos grupos durante los 3 primeros días de evolución.

\section{Discusión}

El presente estudio describe las características, tratamientos recibidos y evolución de los pacientes PT y TG durante el año 2011 en una UCI polivalente de un hospital general. Este es un punto importante a considerar, ya que como se señaló previamente en Chile existen limitados registros de pacientes de trauma en UCI, y los registros internacionales generalmente provienen de centros especializados en la atención del trauma.

Como se ha descrito tradicionalmente en trauma, la mayoría de los pacientes eran hombres jóvenes ${ }^{8,9}$. EL APACHE II, así como la estadía en UCI y VM, fueron similares a las presentadas por el resto de los pacientes de la unidad, y similares a las descritas por otros estudios ${ }^{10}$.

Las lesiones penetrantes representan sólo 15\% de todos los traumas en territorios en que no existe un conflicto bélico o armado, pudiendo ser más frecuentes en zonas de alta violencia ${ }^{3}$. En el presente estudio, $24 \%$ de los pacientes tuvieron un trauma penetrante, lo que es concordante con que el área atendida por el Complejo Asistencial Dr. Sótero del Río presentó un aumento en las lesiones asociadas a delitos durante el $2011^{11}$. Respecto al trauma contuso, al igual que lo descrito por otros autores ${ }^{3}$, el TEC fue la lesión más frecuente. Pero a diferencia de lo descrito en la literatura, en el presente estudio un porcentaje menor de pacientes con trauma contuso tuvo lesiones de tronco (tórax y abdomen), las que se han descrito hasta en $20 \%$ en este tipo de trauma. Sin embargo, debe tenerse presente que este estudio incluyó a pacientes
Tabla 4. Comparación de variables hemodinámicas y de perfusión entre politraumatizados y traumatizados graves

\begin{tabular}{|c|c|c|c|}
\hline Variable & PT & TG & $\mathbf{p}$ \\
\hline PAM $1^{\circ}$ día & $71,3 \pm 13,8$ & $79,5 \pm 17,2$ & 0,03 \\
\hline PAM $2^{\circ}$ día & $73,5 \pm 13,5$ & $85,8 \pm 10,1$ & $<0,001$ \\
\hline PAM $3^{\circ}$ día & $81 \pm 11,9$ & $85,7 \pm 15,6$ & NS \\
\hline Lactato $1^{\circ}$ día & $4,2 \pm 3,9$ & $2,6 \pm 1,9$ & NS \\
\hline Lactato $2^{\circ}$ día & $2,7 \pm 3$ & $3,2 \pm 1$ & NS \\
\hline Lactato $3^{\circ}$ día & $1,9 \pm 0,8$ & $1,6 \pm 0,3$ & NS \\
\hline Hcto $1^{\circ}$ día & $29,3 \pm 8$ & $32,9 \pm 7,7$ & NS \\
\hline Hcto $2^{\circ}$ día & $29,3 \pm 4,9$ & $29,6 \pm 5,6$ & NS \\
\hline Hcto $3^{\circ}$ día & $28,3 \pm 3,5$ & $28,1 \pm 5$ & NS \\
\hline Fluidos $1^{\circ}$ día & $2.569 \pm 1.637$ & $1.771 \pm 894$ & 0,03 \\
\hline Fluidos $2^{\circ}$ día & $2.323 \pm 1.264$ & $1.769 \pm 798$ & NS \\
\hline Fluidos $3^{\circ}$ día & $1.581 \pm 983$ & $1.273 \pm 906$ & NS \\
\hline NA $1^{\circ}$ día & $0,12 \pm 0,1$ & $0,11 \pm 0,1$ & NS \\
\hline NA $2^{\circ}$ día & $0,12 \pm 0,1$ & $0,10 \pm 0,1$ & NS \\
\hline NA $3^{\circ}$ día & $0,12 \pm 0,1$ & $0,05 \pm 0,08$ & NS \\
\hline
\end{tabular}

Presión arterial media (PAM), Hcto (hematocrito), NA (noradrenalina). Comparación de promedios mediante prueba $t$ para muestras independientes, NS (no significativo).

de trauma que ingresaron a la UCI, por lo que podrían existir pacientes con trauma contuso de tronco que hayan fallecido en pabellón o urgencia, y que no están incluidos en este registro.

Llama la atención el alto porcentaje de pacientes que requirieron cirugías o procedimientos, que es mayor a lo reportado por otros estudios realizados en centros especializados en la atención del trauma ${ }^{3,12}$, pero similar a lo reportado en otros centros chilenos ${ }^{13}$. Asimismo, llama la atención el alto porcentaje de pacientes que recibieron transfusión masiva (TM), que habitualmente se ha reportado en 2 a 3\% en los pacientes con trauma civil $^{14,15}$. Respecto a la terapia transfusional, los pacientes recibieron una alta relación PFC: GR $(1,5: 1)$ durante los 2 primeros días, incluso mayor a la usada en pacientes con requerimientos de TM por algunos grupos que plantean estrategias liberales de transfusión ${ }^{6,16}$. El manejo transfusional de los PT y TG es un punto que debe protocolizarse, especialmente porque aún no se ha establecido cuál es la relación óptima entre los distintos he- 
moderivados ${ }^{17}$ y el uso de transfusiones no está exento de complicaciones ${ }^{18}$.

Los aportes moderados de vasoactivos y fluidos que recibieron los pacientes durante los primeros días en UCI, junto con la hiperlactatemia moderada que se depura dentro de las primeras $24 \mathrm{~h}$, apuntan a que estos pacientes fueron reanimados principalmente previo al ingreso, en urgencia y pabellón. Esto es discordante con el balance hídrico acumulado de casi 8 litros al séptimo día. Este es un punto importante de revisar, ya que actualmente existe evidencia de que un aporte excesivo de fluidos puede asociarse a mayor morbi-mortalidad en pacientes $\operatorname{críticos}^{19,20}$. Por otro lado, si bien la mayoría de los pacientes ya habían consolidado su reanimación al tercer día de evolución, sólo un cuarto de ellos estaba recibiendo nutrición en ese momento.

Se ha reportado que el TEC es la causa más frecuente de muerte en pacientes de trauma, seguido por el sangrado no controlado y la disfunción orgánica múltiple-sepsis ${ }^{21,22}$. Dado que un porcentaje importante de los pacientes que mueren por lesiones a nivel del sistema nervioso central lo hacen en el pre hospitalario o en la urgencia, y que la mayoría de los pacientes que mueren por sangrado no controlado lo hacen en pabellón ${ }^{12}$, esto podría explicar que la causa de muerte más frecuente en este estudio fue disfunción orgánica múltiple-sepsis.

Las únicas diferencias encontradas entre PT y TG es que los primeros tuvieron una PAM (presión arterial media) menor y mayores aportes de fluidos durante el primer día en UCI, pero no se encontró ninguna diferencia en morbi-mortalidad. A priori se podría haber esperado que los PT hubieran tenido más complicaciones durante su evolución ya que presentan lesiones en varios sistemas.

La principal limitación de este estudio, es que sólo se incluyeron los pacientes de trauma que ingresaron a la UCI, por lo que no hay datos del pre hospitalario y la urgencia. Si bien el objetivo del presente estudio fue describir las características y evolución de los pacientes de trauma que ingresaron a la UCI, para complementar esta información y mejorar el análisis de los resultados, podría ser interesante realizar un estudio en que se incluyera el período del pre hospitalario y la urgencia.

Tras analizar las características y evolución de los PT y TG que ingresaron a nuestra UCI, se encontraron resultados similares en mortalidad y morbilidad respecto al resto de los pacientes de la unidad y respecto a lo descrito en la literatura. Se deben protocolizar varios aspectos del manejo de estos pacientes en la UCI, especialmente el aporte de fluidos y transfusiones.

\section{Referencias}

1. Kirkpatrick A, Ball C, D'Amours S, Zygun D. Acute resuscitation of the unstable adult trauma patient: bedside diagnosis and therapy. Can J Surg 2008; 51 (1): 57-69.

2. Alexandrescu R, O'Brien S, Lecky F. A review of injury epidemiology in the UK and Europe: some methodological considerations in constructing rates. BMC Public Health 2009; 9: 226-42.

3. Søreide K. Epidemiology of major trauma. Br J of Surg 2009; 96 (7): 697-8.

4. Ministerio de Salud. Guía Clínica Politraumatizado. Santiago: Minsal, 2007.

5. MacKenzie E, Rivara F, Jurkovich G, Nathens A, Frey K, Egleston B, et al. National Evaluation of the Effect of Trauma-Center Care on Mortality. N Engl J Med 2006; 354 (4): 366-78.

6. Holcomb JB, Wade CE, Michalek JE, Chisholm GB, Zarzabal LA, Schreiber MA, et al. Increased plasma and platelet to red blood cell ratios improves outcome in 466 massively transfused civilian trauma patients. Ann Surg 2008; 248 (3): 447-58.

7. Zink KA, Sambasivan CN, Holcomb JB, Chisholm G, Schreiber MA. A high ratio of plasma and platelets to packed red blood cells in the first 6 hours of massive transfusion improves outcomes in a large multicenter study. Am J Surg 2009; 197 (5): 565-70.

8. Pfeifer R, Tarkin I, Rocos B, Pape HC. Patterns of mortality and causes of death in polytrauma patients-Has anything changed? Injury, Int. J. Care Injured 2009; 40 (9): 907-11.

9. Mann E, Baun M, Meininger J, Wade C. Comparison of mortality associated with sepsis in the burn, trauma, and general intensive care unit patient: a systematic review of the literature. Shock 2012; 37 (1): 4-16.

10. Dossett L, Redhage LA, Sawyer R, May A. Revisiting the validity of APACHE II in the trauma ICU: improved risk stratification in critically injured patients. Injury 2009; 40 (9): 993-8.

11. Subsecretaría de Prevención del Delito-Ministerio del Interior y Seguridad Pública: Boletín comunal de Puente Alto Victimización ENUSC y casos policiales de delitos de mayor connotación social 2011. Departamento de Estudios y Estadísticas Subsecretaría de Prevención del Delito. 
12. Kauvar D, Wade C. The epidemiology and modern management of traumatic hemorrhage: US and international perspectives. Critical Care 2005; 9 (Suppl 5): S1-S9.

13. Terán F, Rojas JP, Velásquez M, García G, Henríquez M, Granata P, et al. Ingreso a Unidad de Pacientes Críticos en politraumatizados: frecuencia, tipo de lesiones y sobrevida. Rev Chil Medicina Intensiva 2009; 24 (3): 167.

14. Shaz BH, Dente C, Harris RS, MacLeod JB, Hillyer C. Transfusion management of trauma patients. Anesth Analg 2009; 108 (6): 1760-8.

15. Sihler KC, Napolitano LM. Massive Transfusion New Insights. Chest 2009; 136 (6): 1654-67.

16. Borgman MA, Spinella PC, Perkins JG, Grathwohl KW, Repine T, Beekley AC, et al. The ratio of blood products transfused affects mortality in patients receiving massive transfusions at a combat support hospital. J Trauma 2007; 63 (4): 805-13.

17. Ruiz C, Andresen M. Treatment of Acute Coagulopathy Associated with Trauma. ISRN Critical Care 2013; Article ID 783478, 7 pages, 2013. doi:10.5402/2013/783478.
18. Inaba K, Branco BC, Rhee P, Blackbourne LH, Holcomb JB, Teixeira PG, et al. Impact of plasma transfusion in trauma patients who do not require massive transfusion. J Am Coll Surg 2010; 210 (6): 957-65.

19. Boyd J, Forbes J, Nakada T, Walley K, Russell J. Fluid resuscitation in septic shock: A positive fluid balance and elevated central venous pressure are associated with increased mortality. Crit Care Med 2011; 39 (2): 259-65.

20. The National Heart, Lung, and Blood Institute Acute Respiratory Distress Syndrome (ARDS) Clinical Trials Network. Comparison of Two Fluid-Management Strategies in Acute Lung Injury. N Engl J Med 2006; 354 (24): 2564-75.

21. Sauaia A, Moore F, Moore E, Moser K, Brennan R, Read, et al. Epidemiology of Trauma Deaths: A Reassessment. J Trauma 1995; 38 (2): 185-93.

22. Dutton R, Stansbury L, Leone S, Kramer E, Hess J, Scalea T. Trauma Mortality in Mature Trauma Systems: Are We Doing Better? An Analysis of Trauma Mortality Patterns, 1997-2008. J Trauma 2010; 69 (3): 620-6. 